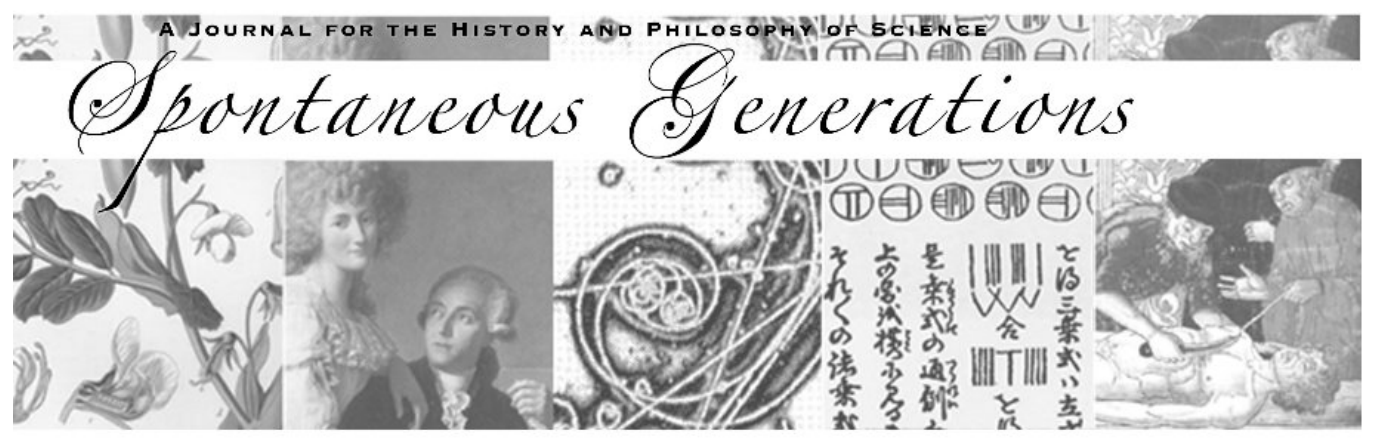

\title{
A Short History of the University of Toronto Collection of Historical Scientific Instruments
}

Author(s): Erich Weidenhammer and Michael Da Silva

Source: Spontaneous Generations: A Journal for the History and Philosophy of Science, Vol. 4, No. 1 (2010) 255-261.

Published by: The University of Toronto

DOI: $10.4245 /$ sponge.v4i1.13802

EDITORIAL OFFICES

Institute for the History and Philosophy of Science and Technology

Room 316 Victoria College, 91 Charles Street West

Toronto, Ontario, Canada M5S $1 \mathrm{~K} 7$

hapsat.society@utoronto.ca

Published online at jps.library.utoronto.ca/index.php/SpontaneousGenerations ISSN 19130465

Founded in 2006, Spontaneous Generations is an online academic journal published by graduate students at the Institute for the History and Philosophy of Science and Technology, University of Toronto. There is no subscription or membership fee. Spontaneous Generations provides immediate open access to its content on the principle that making research freely available to the public supports a greater global exchange of knowledge. 


\title{
OPINIONS
}

\section{A Short History of the University of Toronto Collection of Historical Scientific Instruments*}

\author{
Erich Weidenhammer ${ }^{\dagger}$ and Michael Da Silva ${ }^{\ddagger}$
}

Since the late 1970 s, various attempts have been made to organize the scientific instruments used in research carried out at the University of Toronto into a catalogued, protected, and accessible collection. Unlike other major research universities with which Toronto compares itself, such as Harvard, Yale, Oxford, and Cambridge, to name only a few, these efforts have not been successful. The failure to implement even a modest campus-wide program to safeguard the university's material heritage has had unfortunate consequences. Nevertheless, a great deal of material survives. In the following paper, we examine the circumstances of the historical instruments at the University of Toronto. We argue that this university's scientific instruments are an essential piece of its identity and history. Finally, we propose a practical bottom-up approach through which the current collection can be stabilized and secured (with new instruments added) so that future students can reflect on today's research with the benefit of a rich and well-documented collection.

\section{DECADES OF LOSS}

Efforts towards a University of Toronto science museum, based on a curated collection of historically significant scientific instruments, began in 1977 when the university, acting on the advice of the Institute for the

* Received 4 June 2010.

$\dagger$ Erich Weidenhammer is a PhD candidate at the Institute for the History and Philosophy of Science and Technology at the University of Toronto. His primary research focus is the relationship of chemistry to the medical world of Enlightenment Britain. However, eighteenth-century medical meteorology and its related instrumentation is gaining ground.

$\ddagger$ Michael Da Silva is a J.D. student in the University of Toronto Faculty of Law. His research interests include philosophy of law, social and political philosophy and ethics. $\mathrm{He}$ is particularly interested in bioethics where his interests in philosophy, health law and the history of science all intersect. 
History and Philosophy of Science and Technology (IHPST) struck the Advisory Committee on Historic Scientific Apparatuses. In their report, the committee noted that objects "embody" the history of the development of science in Canada; objects present "a significant, irreplaceable part of the University's heritage, as well as the opportunity to develop their tremendous historic, educational and aesthetic potential for the benefit of both the university community and the general community." The committee also recognized the collection's value to Canadian history enthusiasts and historians of science.

The committee appointed Joy Smith, then a graduate student, to produce a detailed report describing the prospects for a science museum. A massive survey of all surviving scientific instruments was also conducted. With solid institutional backing, Smith and her assistants were able to survey many departments across the university. As part of this survey, Smith and her colleagues contacted numerous instrument-makers, including Leeds and Northrup, Zeiss and Schott, Leitz, and Bausch and Lomb, to collect information about the then-existent collection. Smith's research records and correspondence survive. They make for interesting reading and remain an invaluable guide to the history of scientific research at the University of Toronto.

The nearly two-hundred-page report, presented to the Advisory Committee in August 1978, provides an intriguing glimpse at what might have been. Smith estimated that the collection could be administered by a permanent staff of four-a director/curator and several assistants. In its most ambitions scenario, which emphasized the importance of a centralized collection, the report envisioned a museum housed in the Old Observatory in front of Hart House. This building, erected in 1855, is both the oldest structure on campus and the former home of the oldest scientific institution in the country-the government-sponsored effort to study terrestrial magnetism. A letter from then-University of Toronto President David Strangway, written in 1984, echoes a persistent theme. He wrote: "there are several projects that are more urgent than the conversion of the observatory. I see the project as the sort of initiative that we should keep closely in mind and act on as soon as the opportunity arises." The building still houses the student government as it has since the 1950s.

This effort's most tangible achievement was an instrument catalogue consisting of several thousand file cards. This survives as a sad testament to the speed at which these instruments disappear in the absence of a centralized instrument collection service. The true extent of the losses since 1978 await a full re-cataloguing of the collection but the majority are certainly gone. A survey of various departments that took place in 1999 provides some anecdotes. For instance, a student examining the 
Department of Zoology found only three instruments from Smith's records from a total of over 400, "an abysmal result." Some of the instruments lost since this catalogue was made have been incorporated into other Canadian collections including those of the Royal Ontario Museum, the Ontario Science Centre, the Canadian Science and Technology Museum, the Museum of Health Care at Kingston, and the Niagara Apothecary at Niagara-on-the-Lake. When instruments do end up enriching other public institutions, one can hardly complain given that most of the missing instruments have ended up in the trash.

\section{WHY KEEP INSTRUMENTS?}

If an instrument collection is to thrive, it must attract the support, but, more importantly, the interest of a variety of departments. For an effort that bridges the humanities and the sciences, this is both an opportunity and a challenge. Scholars in the humanities with an academic interest in the material culture of science have very particular reasons for wanting a pool of instruments to study and interpret. To them, instruments are three-dimensional documents providing insight into laboratory and demonstration practices and the specialized skills of the instrument makers or industrial designer. Those interested in a particular area of technology represented in a collection, i.e., radiology or acoustics, might visit the collection-particularly if basic details of the instruments are accessible through an online catalogue. The University of Toronto collection has already been used extensively for such research. Most notably, David Pantalony, an alumnus of the IHPST and current curator of the Canadian Science and Technology Museum, recently published Altered Sensations, a book on the Parisian instrument maker Rudolph Koenig (1832-1901) based on work that began with the university's first-class collection of acoustical instruments.

Anyone who has spent time with such a collection knows that instruments tell stories of their use. Of any group of instruments purchased at the same time or from the same dealer, some will show the wear and tear of heavy use; others will remain pristine. Under lids and covers one can find values scrawled from repeated calibrations, sometimes along with initials. Modifications, repairs, even laboratory-made instruments and prototypes are invaluable to understanding the production of knowledge. Still, this deep and specialized understanding of instruments can seem esoteric to those beyond the humanities.

Those engaged in scientific practice have their own reasons to see that some material evidence is kept and studied. Generally speaking, the prevailing institutional culture in the science departments works against a systematic collections policy in its relentless pursuit of new knowledge. 
The science departments have many priorities and keeping track of old instruments is not necessarily at the top of their list. Equipment quickly becomes obsolete and space is always at a premium. When instruments are kept, it is often due to their personal significance or to the fact that they represent a particular achievement. At worst, when scientists and physicians have interpreted their disciplines they have produced narrowly-conceived accounts of technical achievement which, from the perspective of those trained in the humanities, sacrifice critical rigour and historical interest.

What is needed for a university collection to flourish is a common ground between the humanities and the sciences-a shared understanding of the importance of the artifacts of scientific research. Instruments can serve as important boundary objects. They can help draw historians of science (particularly those without scientific training) closer to the realities of the laboratory which they claim to interpret. They can help correct scientists' teleological notions by showing past work as bounded by vastly different circumstances, assumptions, and values. The cataloguing process, if done thoroughly, will study the provenance of instruments and, in doing so, further illustrate the institutional stories of their use. As the 1978 report noted, instruments can provide science students with "a better understanding of the scientific and social origins of their own disciplines, as well as an appreciation of the close inter-relationships between all disciplines and society." Exhibitions and displays relevant to the institutional history of the departments should be a priority for those who work with the collection. Instruments ought to be interpreted in ways that are interesting, accessible to a wide non-specialist audience, yet still critical in the broad sense of the word.

The instrument collection can be a way for the university to connect with the community. Whether in departmental hallways or a dedicated university science museum, instrument displays can provide the university a way to convey its history as among the oldest and most accomplished places of science in this country. Instruments are, in a way, profoundly central to this university's identity. The University of Toronto cannot trace its roots to the distant past, and, as a federation of colleges, represents no single religious or ethnic tradition. Like its Gothic-revival stone buildings, the pageantry and traditions that it does have are mostly colonial borrowings. The University of Toronto has flourished as a modern public research university and scientific instruments provide excellent evidence of that role. 


\section{MODEST STEPS FORWARD}

Since the 1978 report, there have been many university committees struck in an effort to produce a coherent policy for collecting instruments from the science departments and cataloguing them. These have all failed for a variety of reasons. Typically, there is a substantial cost to the university. One suggestion common to nearly every attempt to create a university-wide policy for safeguarding scientific instruments, from 1978 to the 2002 Standing Committee headed by Physics professor and, then-Vice Dean of Arts and Science, Pekka Sinervo, is the hiring of a full-time curator. This, not to mention the cost of establishing a physical museum, represents a significant long-term cost for the university. Several who have sat on such committees over the years, now emeritus professors or senior faculty, are understandably disillusioned with a process that seems much better at generating reports than results.

Balancing these administrative letdowns is a steady level of enthusiasm from across the campus for these instruments. There are, among many departments, professors, graduate students, and alumni who understand the historical value of these instruments and are working to keep them safe. Physics, psychology, and chemistry all have emeritus professors dedicated to keeping the oldest and most valuable parts of their collections intact. Moreover, there are several humanities departments whose students, experience has shown, can both benefit from and support this project in critical ways and at very little cost.

In the early 2000s, for instance, students in Museum Studies generated a substantial and highly competent collections policy that could serve as the basis for any future museum. This document collected the experience of years of work with the collection and has been cited and studied by subsequent university committees. An online catalogue, the UTMuSI project, led by David Pantalony and Conor Burns, created an online presence for the collection. It continues to attract emails from across the globe. The natural locus for this effort has always been, and will remain, the IHPST. There, graduate students can incorporate these instruments directly into their research for the enrichment of their academic experience and the benefit of their future careers. Recently, with the formation of the University of Toronto Scientific Instrument Collection (UTSIC) project, based at the IHPST, students have begun to find ways to incorporate these instruments into their teaching, and to use them as the basis for exhibits and online essays.

Like the earlier UTMuSI projects and efforts by students at Museum Studies, the UTSIC effort represents a bottom up alternative to the top down initiatives discussed, to little effect, in university committees. Those involved in the project see it as their primary goal to catalogue and 
safeguard existing instruments. In this way, the various instruments in caches around the university can be integrated into a coherent collection upon which a future museum might be established. Perhaps more importantly, once these instruments are identified and tagged as being part of a collection, they are far less likely to be disposed of. To this end, the UTSIC, with the help of the Office of Infrastructure Planning, has acquired a large cataloguing, photography, and storage space that can accommodate instruments that are in immediate danger of being lost.

The central focus of the UTSIC project is the effort to produce an online catalogue. Only such a catalogue can produce a "living" collection in which current information on the whereabouts and nature of all instruments is immediately available. Moreover, an online catalogue makes it possible to draw on the knowledge of experts and aficionados from across the world in identifying the thousands of instruments that must eventually be incorporated into the collection.

There is a further, very promising, opportunity opened up by the online catalogue. Traditionally, such catalogues have been simply searchable lists of instruments containing a basic level of information with accompanying photographs. Such catalogues are primarily of interest to researchers, collectors and hobbyists. However, with the increasing popularity and accessibility of blogging software, it has become possible to incorporate an interpretive/narrative layer which can allow non-experts to engage with the collection and its meaning.

For instance, a homepage, separate from the catalogue, could feature periodically updated short essays on a variety of topics, ranging from collections policy, to departmental history, details of a particular instrument, laboratory culture, and so on. Such essays could provide students in History, HPS, and Museum Studies a way to articulate their interests to a broader audience. Links would be provided, at relevant points in the texts, to particular instruments or types of instruments in the online catalogue. Further links could be provided to photographs and documents in the online databases maintained by the University of Toronto Archives and Records Management Services. Such an approach has the potential to substantially broaden the appeal of the online instrument catalogue and to demonstrate the importance of the instrument collection to the university community. Efforts are underway to produce this interpretively rich catalogue.

Volunteer projects, like UTSIC and its predecessors, provide the university with a cost efficient way to fight inertia prevent the loss of instruments and to provide an organized foundation for future efforts. If the UTSIC manages to find the kind of institutional traction necessary to keep it going over the long term (something that has eluded previous 
efforts) it will represent a major step forward. However, such a volunteer effort will necessarily be limited compared to those a full-time curator could undertake. Graduate students, who constitute the bulk of the volunteers, have many obligations including teaching and their own research and writing. Even when such efforts are supplemented with paid work (research assistantships have been provided by the IHPST, and positions made available by professors applying to the provincial Work-Study program), one could never hope to accomplish what might be possible if the university decided to dedicate significant funds to a curated collection or a museum.

In the meantime, the university can facilitate such efforts by putting in place a basic framework to ensure that valuable instruments are not disposed of carelessly. The creation of a consistent collections policy would be a good first step. At a minimum, one person in each science department should be given the responsibility of keeping track of equipment and of contacting those currently involved with the collection if items are to be moved or discarded. This ought not be an onerous responsibility with grave consequences if mistakes are made. Rather, it should be seen as a modest contribution towards safeguarding the material legacy of scientific work at the university-something from which the whole university stands to benefit.

\section{ACKNOWLEDGEMENTS}

The University of Toronto Scientific Instrument Collection wishes to thank the following for their assistance, financial or otherwise: the Dean's Student Initiatives Fund, the Institute for the History and Philosophy of Science and Technology, the Graduate Students' Union, and the Office of Infrastructure Planning of the Faculty of Arts and Science, all at the University of Toronto.

ERICH WEIDENHAMMER

Apt 1904, 160 Erskine Ave.

Toronto, ON M4P 1 Z3

erich.weidenhammer@utoronto.ca

MICHAEL DA SILVA

304-177 St. George Street

Toronto, ON M5R 2M5

mike.dasilva@utoronto.ca 